\title{
A comparison between the continual reassessment method and $D$-optimum design for dose finding in phase I clinical trials
}

\author{
M. Iftakhar Alam \\ Institute of Statistical Research and Training, University of Dhaka, Dhaka-1000, \\ Bangladesh, e-mail: iftakhar@isrt.ac.bd

\section{SUMMARY} \\ The continual reassessment method is a model-based procedure, described \\ in the literature, used to determine the maximum tolerated dose in phase \\ I clinical trials. The maximum tolerated dose can also be found under \\ the framework of $D$-optimum design, where information is gathered in \\ such a way so that asymptotic variability in the parameter estimates in \\ minimised. This paper investigates the two methods under some realistic \\ settings to explore any potential differences between them. Simulation \\ studies for six plausible dose-response scenarios show that $D$-optimum \\ design can work well in comparison with the continual reassessment \\ method in many cases. The $D$-optimum design is also found to allocate \\ doses from the extremes of the design region to the patients in a trial.
}

Key words: Dose finding studies; Phase I trial; Maximum tolerated dose; Continual reassessment method; $D$-optimum design.

\section{Introduction}

The goal of a phase I trial is to identify the safety, tolerability and pharmacokinetics of a drug. For nontoxic agents, phase I trials are often conducted with healthy volunteers. But for toxic agents, such as those for cancer treatments, phase I trials are conducted among cancer patients in the final stage, for whom standard treatments have failed. Since the benefits from such agents are believed to increase with dose, the highest possible dose is searched for during the development of an agent. However, toxicity also increases with dose. Therefore, the main challenge for those trials is to find a dose with a low chance of causing toxicity to patients. This dose is usually referred to as the maximum tolerated dose (MTD).

Phase I designs can be classified into two broad categories: rule-based 
and model-based. Many rule-based designs only utilise only information from the current cohort in allocating a dose to the next cohort. O'Quigley and Zohar (2006) call these memoryless designs, as the previous information is completely ignored. On the other hand, there are designs which carry information throughout the trial. Designs with memory are mostly modelbased. The essence of the rule-based designs is that they do not assume any parametric dose-response model, but they use instead use pre-specified rules. Some of these designs are based on the up-and-down rule (Dixon and Mood, 1948), where escalation or de-escalation of dose depends on the occurrences of toxicity in the previous cohort. Commonly used rule-based designs include the $3+3$ design, Storer's up-and-down designs (Storer, 1989), pharmacologically-guided dose-escalation design (Collins et al., 1990), accelerated titration designs (Simon et al., 1997) and designs using isotonic regression (Leung and Wang, 2001).

Le Tourneau et al. (2009) report that although several improved statistical methods have been developed in recent years, many current studies still use a traditional $3+3$ design because of its simplicity. The $3+3$ design is often used since the specific issues to be achieved in phase I trials are not stated clearly. According to O'Quigley et al. (1990), a phase I design should aim to: (1) minimise the number of under-treated patients and the number of over-treated patients; (2) minimise the number of patients needed to complete the study; and (3) rapidly escalate the dose in the absence of toxicity or rapidly de-escalate the dose in the presence of an unacceptable level of toxicity. It is possible in a $3+3$ design to come to a conclusion by using only a few patients, but Reiner et al. (1999) and Lévy et al. (2001) have shown that the probability of an incorrect recommendation for the MTD is very high for this design.

Model-based designs are alternatives to rule-based designs. They assume a parametric model to establish the dose-response relationship. Such designs select a dose level that produces a target probability of toxicity using all of the accrued data in a trial. These designs are usually implemented under the Bayesian framework, as the sample size remains small at the early stages of a trial. Common model-based designs include the continual reassessment method (O'Quigley et al., 1990), escalation with overdose control (Babb et al., 1998) and others. At each stage of an adaptive trial, the continual reassessment method (CRM) allocates a dose to a patient such that the estimate of probability of toxicity at that dose is closest to a target toxicity rate. 
The theory of optimal design can also help in the search for the MTD. For instance, we may allocate doses to the patients in such a way that the determinant of the Fisher information matrix (FIM) is maximised. The maximisation is carried out over a set of available doses and this criterion of design optimality is known as the $D$-criterion in the literature. The CramérRao inequality tells us that the covariance matrix of the parameter estimators is greater than, and asymptotically approaches, the inverse of the FIM. Therefore, by maximising the determinant of the FIM through the $D$-criterion, we can minimise the asymptotic lower bound for the variance of the estimated model parameters. This paper investigates the properties of the two designs under the same settings.

The organisation of the paper is as follows. Section 2 describes the two dose finding methods to be compared. The settings of the simulation and the major findings are discussed in Sections 3 and 4, respectively. Finally, the conclusions are stated in Section 5.

\section{Methods}

Assume that $d$ ordered doses $\mathcal{X}=\left\{x^{(1)}, \ldots, x^{(d)}\right\}$ are available for an experimental drug based on preclinical studies, and we want to determine the MTD to be used in the next phase. A good design should be able to identify the MTD accurately without exposing many patients to either subtherapeutic or toxic doses. The following sections describe the continual reassessment method and the $D$-optimum design apprach to find the MTD.

\subsection{Continual Reassessment Method}

The continual reassessment method is a model-based procedure for finding the MTD in phase I clinical trials (O'Quigley et al., 1990). The design aims to reduce the number of patients at subtherapeutic doses and to obtain a more accurate estimate of the MTD. The method employs parametric models, such as the hyperbolic tangent model, logistic model or power model to characterise the dose-response relationship. Investigators sometimes use a one-parameter logistic model with the idea that it would require fewer patients to obtain a precise estimate of the unknown parameter. However, such a model may not be flexible enough to depict the dose-response relationship accurately. Therefore, we plan to use a two-parameter logistic model, as shown below, so that the relationship can be established more accurately. 


$$
\psi(x, \boldsymbol{\vartheta})=\frac{\exp \left(\vartheta_{1}+\vartheta_{2} x\right)}{1+\exp \left(\vartheta_{1}+\vartheta_{2} x\right)}
$$

where $\boldsymbol{\vartheta}=\left(\vartheta_{1}, \vartheta_{2}\right)$ is the vector of dose-response parameters and $x$ is the dose given to a patient. If we are at the $k$ th stage in a trial, this means that $k$ patients have been treated with different doses from $\mathcal{X}$. Let $\boldsymbol{x}$ be a $k \times 1$ dose vector with components $x_{l}$ and let $\boldsymbol{r}$ be a $k \times 1$ outcome vector with $r_{l}$ as the $l$ th row $(l=1, \ldots, k)$ representing the toxic outcomes obtained from a patient. Then the likelihood function at stage $k$ can be written as

$$
L_{k}(\boldsymbol{\vartheta} \mid \boldsymbol{x}, \boldsymbol{r}) \propto \prod_{l=1}^{k}\left\{\psi\left(x_{l}, \boldsymbol{\vartheta}\right)\right\}^{r_{l}}\left\{1-\psi\left(x_{l}, \boldsymbol{\vartheta}\right)\right\}^{1-r_{l}} .
$$

Since maximum likelihood estimates are not possible until sufficient information is available, we employ a Bayesian approach to estimate the dose-response parameters $\boldsymbol{\vartheta}$. The posterior means of the components of $\boldsymbol{\vartheta}=\left(\vartheta_{1}, \vartheta_{2}\right)$ at the $k$ th stage are obtained as

$$
\hat{\vartheta}_{i k}=\frac{\int_{\Theta} \vartheta_{i} g(\boldsymbol{\vartheta}) L_{k}(\boldsymbol{\vartheta} \mid \boldsymbol{x}, \boldsymbol{r}) d \boldsymbol{\vartheta}}{\int_{\Theta} g(\boldsymbol{\vartheta}) L_{k}(\boldsymbol{\vartheta} \mid \boldsymbol{x}, \boldsymbol{r}) d \boldsymbol{\vartheta}}, \quad i=1,2,
$$

where $\Theta$ is the parameter space and $g(\boldsymbol{\vartheta})$ is the prior distribution of the parameters. A bivariate uniform density is assumed for the parameters. The choice of $u_{1}<\vartheta_{1}<u_{2}$ and $u_{3}<\vartheta_{2}<u_{4}$ gives a restricted parameter space as $\tilde{\Theta}=\left\{\boldsymbol{\vartheta}: u_{1}<\vartheta_{1}<u_{2}, \quad u_{3}<\vartheta_{2}<u_{4}\right\}$ so that

$$
g(\boldsymbol{\vartheta})=\frac{1}{\left(u_{2}-u_{1}\right)\left(u_{4}-u_{3}\right)}, \quad \boldsymbol{\vartheta} \in \tilde{\Theta} .
$$

Then the probability of toxicity at the end of stage $k$ is updated at each dose as

$$
\hat{\psi}_{i k}=\psi\left(x^{(i)}, \hat{\vartheta}_{k}\right), \quad i=1,2, \ldots, d .
$$

That dose chosen for the next patient is such that the absolute difference between the updated estimate of the probability of toxicity and the target toxicity rate $\gamma$ is minimum. That is,

$$
x_{k+1}=\arg \min _{x \in \mathcal{X}}\left|\psi\left(x, \hat{\vartheta}_{k}\right)-\gamma\right| .
$$


The trial continues until a fixed sample size $n$ is achieved and the MTD is determined as the dose that would be allocated to patient $n+1$ if he were in the trial. The typical values for $\gamma$ are $0.2,0.25,0.3$, or 0.33 . There were some difficulties perceived by clinicians regarding the original version of the CRM. These include starting with the dose which is the best prior guess of the MTD, incorporating prior information regarding the model parameters and the numerical integration necessary in the implementation of the method.

O'Quigley and Shen (1996) proposed a new version of the CRM based on the classical likelihood approach of parameter estimation known as the continual reassessment maximum likelihood (CRML) method. The CRML appears to deal with the above three difficulties. Since initially the new design uses an up-and-down design which starts with the lowest dose, it does not require the best prior guess of the MTD to start with. Also, as the design is based on the frequentist approach, it ignores any prior information regarding the parameters. The operating characteristics of the two methods are very similar. Although simulation studies show some minor differences in dose allocation during the trials, the final recommendations are almost the same. As the likelihood equation has no solution until a toxic outcome is observed, the CRML can be applied only after the occurrence of such an outcome. To overcome this, a suggestion is to use initially either a standard up-and-down procedure or the CRM until a toxic outcome is observed, after which dose allocation can be based on the CRML.

In the original version of the CRM, many patients are likely to be exposed to high toxicity because of dose skipping dose. Various modifications have been suggested to make it safer, as detailed in Le Tourneau et al. (2009). Two of these are considered in this paper: (1) treating the first patient at the lowest dose level (Korn et al., 1994); and (2) increasing the dose by only one pre-specified level at a time (Goodman et al., 1995).

\section{2. $\quad$-optimum Design}

The $D$-optimum design approach allocates to the patients in a trial those doses which contribute most to the efficient estimation of the dose-response parameters. If the dose-response model is non-linear in the parameters, the FIM will depend on the unknown parameters. Therefore, in searching for the optimal dose, we need to assume prior values of the model parameters, and consequently the design is known as locally optimal (Chernoff, 1953). The estimates of the parameters obtained at each stage of an adaptive trial 
can serve as prior for the next stage. As more accurate estimates of the parameters are expected as a trial progresses, we can expect more appropriate doses to be allocated to the patients as well.

Assume that we are at the $k$ th stage in a trial. The $k$ patients have then received doses from $\mathcal{X}$ and are represented as $\boldsymbol{\xi}_{k}=\left\{x_{1}, x_{2}, \ldots, x_{k}\right\}$. Also, based on the dose-response outcomes so far, the posterior estimate $\hat{\boldsymbol{\vartheta}}_{k}$ of the parameters is available in the way explained earlier. Now let us define

$$
\boldsymbol{M}\left(x \mid \boldsymbol{\xi}_{k}, \hat{\boldsymbol{\vartheta}}_{k}\right)=\frac{k}{k+1} \boldsymbol{M}\left(\boldsymbol{\xi}_{k}, \hat{\boldsymbol{\vartheta}}_{k}\right)+\frac{1}{k+1} \boldsymbol{I}\left(x, \hat{\boldsymbol{\vartheta}}_{k}\right),
$$

where $\boldsymbol{M}\left(\boldsymbol{\xi}_{k}, \hat{\boldsymbol{\vartheta}}_{k}\right)=\sum_{l=1}^{k} \boldsymbol{I}\left(x_{l}, \hat{\boldsymbol{\vartheta}}_{k}\right)$ and $\boldsymbol{I}\left(x_{l}, \hat{\boldsymbol{\vartheta}}_{k}\right)$ is the Fisher information matrix for a patient who received the dose $x_{l}$. For the dose-response model introduced in (1), the likelihood function for a single patient at dose $x_{l}$ is

$$
L\left(\boldsymbol{\vartheta} \mid x_{l}, r_{l}\right)=\left(\psi_{l}\right)^{r_{l}}\left(1-\psi_{l}\right)^{1-r_{l}},
$$

and so the log-likelihood function can be written as

$$
l\left(\boldsymbol{\vartheta} \mid x_{l}, r_{l}\right)=r_{l} \log \left(\psi_{l}\right)+\left(1-r_{l}\right) \log \left(1-\psi_{l}\right) .
$$

Then the Fisher information matrix for a patient can be obtained as

$$
\boldsymbol{I}\left(x_{l}, \boldsymbol{\vartheta}\right)=\left[\begin{array}{cc}
\psi_{l}\left(1-\psi_{l}\right) & x_{l} \psi_{l}\left(1-\psi_{l}\right) \\
x_{l} \psi_{l}\left(1-\psi_{l}\right) & x_{l}^{2} \psi_{l}\left(1-\psi_{l}\right)
\end{array}\right] \text {. }
$$

Atkinson et al. (2014) discuss the construction of optimal experimental designs based on augmented FIMs. We can select the dose $x_{k+1}$ for the next patient such that

$$
x_{k+1}=\arg \max _{x \in \mathcal{X}} \Phi_{D}\left\{\boldsymbol{M}\left(x \mid \boldsymbol{\xi}_{k}, \hat{\vartheta}_{k}\right)\right\}
$$

where $\Phi_{\mathrm{D}}\{\boldsymbol{M}\}=|\boldsymbol{M}|$. A trial is continued until the fixed sample size $n$ is achieved. Here the estimated dose-toxicity curve based on the parameter estimates at the last stage are used to find the MTD. The dose for which the absolute difference between the estimated probability of toxicity and the target toxicity rate is minimum is taken as the MTD. That is, the same rule is followed for MTD selection in both of the designs. 


\section{Setup for Simulations}

Six plausible dose-response scenarios are assumed for the simulation study: see Figure 1. The set of six available doses in each scenario is $\mathcal{X}=\{1,3,5, \ldots$, $11\}$. The scenarios differ only in shape, with a sharp increase of toxicity in Scenario 1 and a slow increase in Scenario 6. It is assumed that the target toxicity rate $\gamma$ is 0.33 . Doses 3, 5, 7 and 11 are the respective true MTDs in the first four scenarios. These are the doses at which the probability of toxicities is less than or equal to the target rate. More specifically, the probability of toxicities at these doses are $0.32,0.32,0.33$ and 0.32 , respectively. The last two scenarios are slightly different from the first four in the sense that the target toxicity rate corresponds to a dose that lies in between two available doses. The true MTD is 6 in Scenario 5, and it lies between the available doses 5 and 7 , which have respective probabilities of toxicity of 0.24 and 0.43. Since dose 6 is not available in $\mathcal{X}$, we assume 5 as the true MTD. Similarly, in Scenario 6, the true MTD 10 lies between 9 and 11. These doses have respective probabilities of toxicity of 0.28 and 0.39 . Here we treat dose 9 as the true MTD. In real trials, we may start with a set of doses where none of the doses have a probability of toxicity exactly or approximately equal to the target. The last two scenarios are considered in order to observe the behaviour of the designs in such situations. For the Bayesian estimation, we consider a bivariate uniform distribution for the dose-response parameters $\boldsymbol{\vartheta}$. We consider $\tilde{\Theta}=\left\{\boldsymbol{\vartheta}:-4.3<\vartheta_{1}<-2.3,0<\vartheta_{2}<1\right\}$, a single parameter space for all of the scenarios. The parameter space has been found to allow a wide range of dose-response scenarios, including those assumed for the simulation study. The response after receipt of a dose by a patient is generated from the binomial distribution.

Each trial starts with the lowest dose of $1 \mathrm{mg} / \mathrm{kg}$-body weight applied to a patient. After the outcomes are received, the dose-response parameters are estimated, as described in Section 2.1.. Then we select a dose following the dose-optimisation criterion either in the CRM or in $D$. The selected dose is applied to a new patient. After the outcomes from the second patient are received, the dose-response parameters are estimated and a dose is chosen for the third patient. The process continues until the trial reaches the maximum number of patients $n$, after which the MTD is determined. The designs are investigated for various values of $n$, including 15, 20, 25 and 30. Each of the scenarios is investigated through 2000 simulated trials using self-written

code in $R$. The program takes on average 30 minutes to run the simulations for a scenario on a computer with Core i3 processor and 4 GB RAM. 

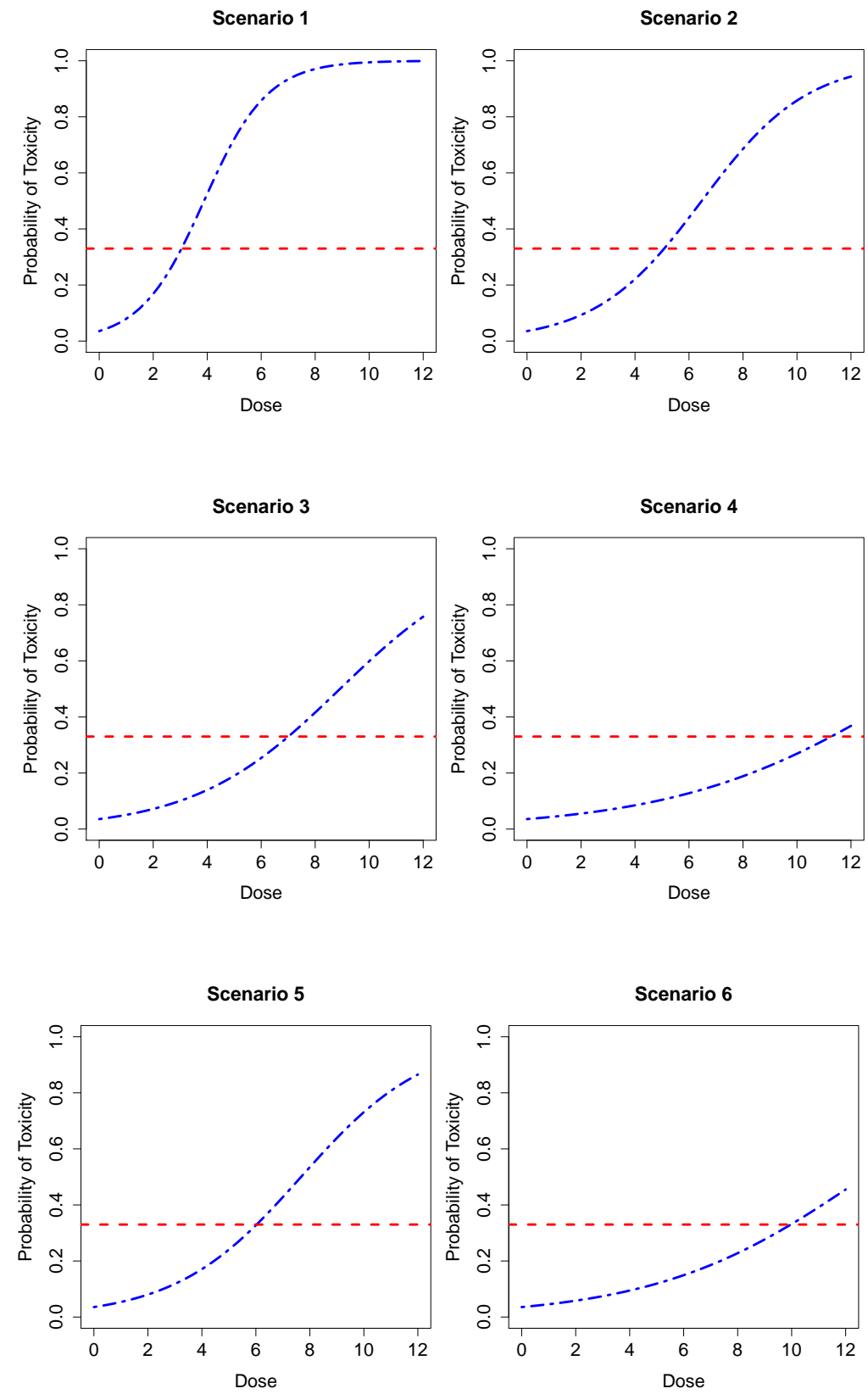

Figure 1. Dose-response scenarios for simulation study. The respective parameter values are: Scenario $1, \boldsymbol{\vartheta}=(-3.3,0.85)$; Scenario $2, \boldsymbol{\vartheta}=(-3.3,0.51)$; Scenario $3, \boldsymbol{\vartheta}=(-3.3,0.37)$; Scenario $4, \boldsymbol{\vartheta}=(-3.3,0.23)$; Scenario $5, \boldsymbol{\vartheta}=(-3.3,0.43)$; and Scenario $6, \boldsymbol{\vartheta}=(-3.3,0.26)$. The dotted horizontal line indicates the target toxicity rate. 


\section{Simulation Findings}

Scenario 1 has the dose 3 as the true MTD and it is selected in $92.6 \%$ of the trials by the CRM: see Table 1. This dose is selected in $96.0 \%$ of the trials by $D$-optimum design. As $n$ increases, the correct identification of the MTD improves for both of the designs. As a whole, $D$-optimum design identifies the MTD more accurately than the CRM in this scenario. However, the percentage of patients that receive the true MTD during the trials is much greater in the case of CRM than in that of $D$-optimum design. For instance, when $n=20$, the CRM treats $80.3 \%$ of the patients at the MTD, compared with $23.7 \%$ for $D$-optimum design. Many patients are treated at the higher doses during the trials with $D$-optimum design. A smaller number of trials recommend a highly toxic dose as the MTD in $D$-optimum design. Note that the row sums in the tables may not be exactly equal to 100 , due to rounding.

Table 1. The percentage of times the doses are identified as the MTD and the percentage of patients treated at those doses during the trials (in parentheses)

for Scenario 1.

\begin{tabular}{cccccccc}
\hline$n$ & Design & \multicolumn{5}{c}{ Dose } \\
\cline { 3 - 7 } & & 1 & $\mathbf{3}$ & 5 & 7 & 9 & 11 \\
\hline 15 & CRM & $0.8(6.8)$ & $92.6(75.3)$ & $6.7(15.7)$ & $0.0(2.1)$ & $0.0(0.0)$ & $0.0(0.1)$ \\
& $D$ & $0.1(24.1)$ & $96.0(24.2)$ & $3.9(21.6)$ & $0.0(25.1)$ & $0.0(4.4)$ & $0.0(0.5)$ \\
20 & CRM & $0.5(5.3)$ & $96.2(80.3)$ & $3.3(12.6)$ & $0.1(1.7)$ & $0.0(0.0)$ & $0.0(0.1)$ \\
& $D$ & $0.4(24.8)$ & $97.5(23.7)$ & $2.1(23.0)$ & $0.0(24.8)$ & $0.0(3.4)$ & $0.0(0.4)$ \\
25 & CRM & $0.5(4.3)$ & $96.7(84.1)$ & $2.9(10.4)$ & $0.0(1.2)$ & $0.0(0.0)$ & $0.0(0.1)$ \\
& $D$ & $0.3(25.5)$ & $98.5(23.5)$ & $1.3(23.4)$ & $0.0(24.5)$ & $0.0(2.8)$ & $0.0(0.4)$ \\
30 & CRM & $0.6(3.7)$ & $98.0(86.3)$ & $1.5(8.9)$ & $0.0(1.0)$ & $0.0(0.0)$ & $0.0(0.1)$ \\
& $D$ & $0.6(26.7)$ & $99.0(22.1)$ & $0.5(25.1)$ & $0.0(23.4)$ & $0.0(2.4)$ & $0.0(0.3)$ \\
\hline
\end{tabular}

Dose 5 is the true MTD in Scenario 2 and it lies towards the middle of the dose region. Notice that $D$-optimum design outperforms the CRM in MTD selection, as shown in Table 2. Unlike the previous scenario, here appreciable differences exist in the MTD selection by the designs. On most occasions $D$-optimum design reduces the occurrence of highly toxic doses as the MTD. The design also limits the appearance of lower doses as the MTD. Although many patients are exposed to the highly toxic doses during the progress of trials in $D$-optimum design, this is not the case in the other design.

Scenario 3 has dose 7 as the true MTD. D-optimum design selects this dose more accurately than the CRM. Identification of the MTD increases 
Table 2. The percentage of times the doses are identified as the MTD and the percentage of patients treated at those doses during the trials (in parentheses)

for Scenario 2.

\begin{tabular}{cccccccc}
\hline$n$ & Design & \multicolumn{7}{c}{ Dose } \\
\cline { 3 - 8 } & & 1 & 3 & $\mathbf{5}$ & 7 & 9 & 11 \\
\hline \multirow{2}{*}{15} & CRM & $0.0(6.7)$ & $24.0(28.0)$ & $63.0(45.4)$ & $11.9(16.1)$ & $1.1(1.7)$ & $0.2(2.3)$ \\
& $D$ & $0.0(8.2)$ & $17.0(29.8)$ & $73.4(8.1)$ & $9.0(26.8)$ & $0.6(12.5)$ & $0.0(14.6)$ \\
20 & CRM & $0.0(5.0)$ & $21.7(25.8)$ & $67.3(50.4)$ & $10.9(15.6)$ & $0.3(1.5)$ & $0.0(1.8)$ \\
& $D$ & $0.0(6.3)$ & $12.7(31.8)$ & $77.4(6.2)$ & $9.9(28.7)$ & $0.1(11.9)$ & $0.0(15.1)$ \\
25 & CRM & $0.0(4.0)$ & $21.0(27.1)$ & $70.7(52.9)$ & $8.3(13.6)$ & $0.1(1.1)$ & $0.0(1.3)$ \\
& $D$ & $0.0(5.2)$ & $10.2(33.0)$ & $81.9(5.1)$ & $7.9(29.1)$ & $0.0(11.5)$ & $0.0(16.0)$ \\
30 & CRM & $0.0(3.3)$ & $17.7(24.3)$ & $75.1(56.3)$ & $7.2(13.8)$ & $0.0(1.0)$ & $0.0(1.3)$ \\
& $D$ & $0.0(4.4)$ & $8.5(33.9)$ & $84.2(4.4)$ & $7.4(29.8)$ & $0.0(11.3)$ & $0.0(16.2)$ \\
\hline
\end{tabular}

as $n$ increases: see Table 3. Not many trials recommend either a lower or higher dose as the MTD in D-optimum design, compared with the case of the CRM. However, many patients are exposed to toxic doses during the trials with $D$-optimum design.

Table 3. The percentage of times the doses are identified as the MTD and the percentage of patients treated at those doses during the trials (in parentheses) for Scenario 3.

\begin{tabular}{cccccccc}
\hline \multirow{2}{*}{$n$} & Design & \multicolumn{7}{c}{ Dose } \\
\cline { 3 - 8 } & & 1 & 3 & 5 & $\mathbf{7}$ & 9 & 11 \\
\hline \multirow{2}{*}{15} & CRM & $0.0(6.7)$ & $5.1(10.4)$ & $31.3(31.7)$ & $41.3(29.8)$ & $19.0(11.2)$ & $3.5(10.3)$ \\
& $D$ & $0.0(10.7)$ & $0.9(19.6)$ & $37.0(10.1)$ & $45.5(17.5)$ & $14.3(12.6)$ & $2.4(29.6)$ \\
20 & CRM & $3.6(5.0)$ & $3.6(9.8)$ & $30.2(31.4)$ & $50.3(33.8)$ & $14.3(11.8)$ & $1.7(8.1)$ \\
& $D$ & $0.0(8.7)$ & $0.1(21.6)$ & $27.0(8.3)$ & $58.5(18.8)$ & $12.7(10.6)$ & $1.8(32.0)$ \\
25 & CRM & $0.0(4.0)$ & $2.4(8.7)$ & $26.9(30.2)$ & $54.7(37.3)$ & $15.3(13.1)$ & $0.8(6.7)$ \\
& $D$ & $0.0(7.3)$ & $0.2(22.8)$ & $22.6(7.0)$ & $63.4(20.1)$ & $12.5(9.1)$ & $1.4(33.6)$ \\
30 & CRM & $0.0(3.3)$ & $1.9(7.3)$ & $26.6(30.2)$ & $59.1(40.9)$ & $12.1(12.7)$ & $0.3(5.5)$ \\
& $D$ & $0.0(6.5)$ & $0.0(23.6)$ & $18.3(6.2)$ & $70.7(20.7)$ & $10.3(8.1)$ & $0.7(35.0)$ \\
\hline
\end{tabular}

As presented in Table 4, the true MTD is selected in $59.1 \%$ of the trials by the CRM in Scenario 4. The corresponding figure for $D$-optimum design is $23 \%$. As in the previous scenarios, the percentage of correct identification increases as $n$ increases. Here the CRM performs well in comparison with the other design.

In Scenario 5, the target toxicity rate lies midway between two available doses. Dose 5 has a probability of toxicity less than the target and dose 7 has a probability of toxicity greater than the target. As a consequence, a dosefinding algorithm will tend to select these doses as the MTD. As indicated in Table 5 , dose 5 is selected in the majority of the trials. This is due to the fact that the toxicity rate at dose 5 is closer to the target than that of 
Table 4. The percentage of times the doses are identified as the MTD and the percentage of patients treated at those doses during the trials (in parentheses)

for Scenario 4.

\begin{tabular}{cccccccc}
\hline$n$ & Design & \multicolumn{7}{c}{ Dose } \\
\cline { 3 - 7 } & & 1 & 3 & 5 & 7 & 9 & $\mathbf{1 1}$ \\
\hline \multirow{2}{*}{15} & CRM & $0.0(6.7)$ & $0.9(4.4)$ & $4.7(15.5)$ & $12.0(17.3)$ & $23.3(12.4)$ & $59.1(43.7)$ \\
& $D$ & $0.0(22.1)$ & $0.0(5.0)$ & $24.1(19.4)$ & $10.4(5.0)$ & $42.6(18.3)$ & $23.0(30.2)$ \\
20 & CRM & $0.0(5.0)$ & $0.5(3.7)$ & $3.0(12.0)$ & $10.6(15.7)$ & $21.3(14.2)$ & $64.8(49.4)$ \\
& $D$ & $0.0(21.6)$ & $0.0(4.8)$ & $15.8(19.3)$ & $9.8(4.7)$ & $32.6(19.0)$ & $41.9(30.5)$ \\
25 & CRM & $0.0(5.0)$ & $0.5(3.7)$ & $3.0(12.0)$ & $10.6(15.7)$ & $21.3(14.2)$ & $64.8(49.4)$ \\
& $D$ & $0.0(21.3)$ & $0.0(4.9)$ & $17.4(18.9)$ & $6.9(4.9)$ & $32.6(18.6)$ & $43.2(31.3)$ \\
30 & CRM & $0.0(3.3)$ & $0.1(2.7)$ & $1.5(9.4)$ & $6.4(13.5)$ & $23.8(17.8)$ & $68.4(53.2)$ \\
& $D$ & $0.0(21.3)$ & $0.0(4.8)$ & $18.9(18.9)$ & $6.5(4.7)$ & $30.8(18.7)$ & $43.8(31.7)$ \\
\hline
\end{tabular}

at dose 7 . Notice that $D$-optimum design performs well in comparison with the other design in this scenario.

Table 5. The percentage of times the doses are identified as the MTD and the percentage of patients treated at those doses during the trials (in parentheses)

for Scenario 5 .

\begin{tabular}{cccccccc}
\hline \multirow{2}{*}{$n$} & Design & \multicolumn{7}{c}{ Dose } \\
\cline { 3 - 8 } & & 1 & 3 & $\mathbf{5}$ & 7 & 9 & 11 \\
\hline \multirow{2}{*}{15} & CRM & $0.0(6.7)$ & $11.5(16.8)$ & $50.1(39.4)$ & $32.4(26.8)$ & $5.6(5.5)$ & $0.5(4.9)$ \\
& $D$ & $0.0(8.5)$ & $4.5(25.0)$ & $57.2(8.3)$ & $34.0(22.1)$ & $4.0(12.1)$ & $0.5(24.1)$ \\
20 & CRM & $0.0(5.0)$ & $9.6(16.1)$ & $51.9(42.6)$ & $35.1(27.9)$ & $3.4(4.9)$ & $0.1(3.6)$ \\
& $D$ & $0.0(6.5)$ & $2.0(27.0)$ & $55.8(6.4)$ & $40.1(23.5)$ & $2.0(10.5)$ & $0.1(26.2)$ \\
25 & CRM & $0.0(4.0)$ & $6.4(13.8)$ & $55.9(45.2)$ & $35.8(29.3)$ & $2.0(4.7)$ & $0.0(3.0)$ \\
& $D$ & $0.0(5.2)$ & $1.5(28.1)$ & $57.1(5.1)$ & $39.9(24.6)$ & $1.6(9.0)$ & $0.0(28.0)$ \\
30 & CRM & $0.0(3.3)$ & $5.1(12.6)$ & $55.8(45.6)$ & $37.9(31.3)$ & $1.3(4.5)$ & $0.0(2.7)$ \\
& $D$ & $0.0(4.6)$ & $0.9(28.6)$ & $56.8(4.6)$ & $41.4(24.8)$ & $1.0(8.5)$ & $0.0(29.0)$ \\
\hline
\end{tabular}

Unlike Scenario 5, where the true MTD lies in the middle of the dose region, in Scenario 6 it lies in the upper part of the dose region. The MTD lies midway between doses 9 and 11. Most of the trials recommend these doses as the MTD in the simulations: see Table 6. The CRM performs well in comparison with $D$-optimum design. The CRM works well in Scenario 4 as well. These two scenarios are similar; the only difference is that in one we have a dose in the set of available doses with probability of toxicity very close to the target, while in the other, we do not have such a dose - instead it lies between two available doses.

Table 7 shows the downward biases in the estimation of probability of toxicity at the MTD. These indicate that apart from the correct identification, the designs choose lower doses as the MTD more often than unacceptably toxic doses. Apart from a few exceptions, bias decreases for the 
Table 6. The percentage of times the doses are identified as the MTD and the percentage of patients treated at those doses during the trials (in parentheses) for Scenario 6 .

\begin{tabular}{cccccccc}
\hline$n$ & Design & \multicolumn{7}{c}{ Dose } \\
\cline { 3 - 7 } & & 1 & 3 & 5 & 7 & $\mathbf{9}$ & 11 \\
\hline \multirow{2}{*}{15} & CRM & $0.0(6.7)$ & $1.6(5.5)$ & $7.6(17.4)$ & $20.0(20.9)$ & $30.0(15.1)$ & $40.9(34.5)$ \\
& $D$ & $0.0(19.9)$ & $0.1(7.4)$ & $22.8(17.5)$ & $20.0(7.3)$ & $39.7(16.9)$ & $17.5(31.0)$ \\
20 & CRM & $0.0(5.0)$ & $0.9(4.6)$ & $6.1(15.4)$ & $17.7(20.6)$ & $35.3(19.0)$ & $40.2(35.4)$ \\
& $D$ & $0.0(18.8)$ & $0.0(8.3)$ & $15.3(16.6)$ & $21.8(7.6)$ & $34.2(16.8)$ & $28.8(31.9)$ \\
25 & CRM & $0.0(4.0)$ & $0.6(3.5)$ & $5.0(13.4)$ & $16.3(20.2)$ & $35.7(21.0)$ & $42.3(38.0)$ \\
& $D$ & $0.00(18.4)$ & $0.0(8.3)$ & $15.7(16.3)$ & $16.0(7.9)$ & $37.6(16.3)$ & $29.8(32.9)$ \\
30 & CRM & $0.0(3.3)$ & $0.2(3.0)$ & $3.0(11.9)$ & $15.0(19.2)$ & $38.6(23.6)$ & $43.6(39.1)$ \\
& $D$ & $0.0(18.2)$ & $0.0(8.3)$ & $15.5(16.1)$ & $14.7(7.9)$ & $38.8(16.2)$ & $31.1(33.3)$ \\
\hline
\end{tabular}

designs as $n$ increases. $D$-optimal design produces less bias than the CRM in Scenarios 1, 2 and 5. The designs are quite comparable in terms of bias in Scenario 3. These are the scenarios where the $D$-optimal design performs better than the other design. It was noted above that the CRM performs well in Scenarios 4 and 6, and this is again supported by the estimates of bias.

Table 7. Bias in the estimation of probability of toxicity at the recommended MTD.

\begin{tabular}{cccccc}
\hline Scenario & Design & \multicolumn{4}{c}{$n$} \\
\cline { 3 - 6 } & & 15 & 20 & 25 & 30 \\
\hline \multirow{2}{*}{1} & CRM & -0.02218 & -0.02337 & -0.01907 & -0.02387 \\
& $D$ & -0.00619 & -0.00693 & -0.00790 & -0.00599 \\
2 & CRM & -0.07610 & -0.02097 & -0.01972 & -0.02038 \\
& $D$ & -0.00997 & -0.00371 & -0.00478 & -0.00239 \\
3 & CRM & -0.01266 & -0.01387 & -0.01049 & -0.01481 \\
& $D$ & -0.02755 & -0.01842 & -0.01304 & -0.01002 \\
4 & CRM & -0.06304 & -0.05949 & -0.05259 & -0.04559 \\
& $D$ & -0.13567 & -0.11881 & -0.11019 & -0.10865 \\
5 & CRM & -0.01695 & -0.01691 & -0.01829 & -0.01738 \\
& $D$ & -0.01541 & -0.00799 & -0.01058 & -0.01029 \\
6 & CRM & -0.03746 & -0.03007 & -0.02715 & -0.02185 \\
& $D$ & -0.10741 & -0.08984 & -0.07872 & -0.07496 \\
\hline
\end{tabular}

\section{Conclusion}

This paper attempts to explore the behaviour of the CRM and $D$-optimum design under some realistic settings. $D$-optimum design often fails to attract interest due to the fear that efficiency in parameter estimation may not be achieved at early stages of a trial, as a small number of patients are usually 
engaged. But we have seen that $D$-optimum design can identify the MTD more accurately than the CRM in many of the scenarios. The design cannot identity the MTD as accurately as the CRM when the MTD lies in the upper dose region. This is perhaps due to the fact that the design cannot learn much about the dose-response relationship, as it fails to allocate much higher doses to the patients due to the lack of availability of such doses. As indicated by the dose allocation, we have found $D$-optimum design to allocate doses from the extremes of the design region. Although the results are produced here for the logistic model, we believe that use of other models will produce similar results. As a whole, the findings from the simulation study have the potential to motivate clinical investigators to consider the use of $D$-optimum design in phase I clinical trials.

\section{REFERENCES}

Atkinson A.C., Fedorov V.V., Herzberg A.M., Zhang R. (2014): Elemental information matrices and optimal experimental design for generalized regression models. Journal of Statistical Planning and Inference 144(1): 81-91.

Babb J., Rogatko A., Zacks S. (1998): Cancer phase I clinical trials: Efficient dose escalation with overdose control. Statistics in Medicine 17(10): 1103-1120.

Chernoff H. (1953): Locally optimal designs for estimating parameters. The Annals of Mathematical Statistics 24(4): 586-602.

Collins J.M., Grieshaber C.K., Chabner B.A. (1990): Pharmacologically guided phase I clinical trials based upon preclinical drug development. Journal of the National Cancer Institute 82(16): 1321-1326.

Dixon W.J., Mood A.M. (1948): A method for obtaining and analyzing sensitivity data. Journal of the American Statistical Association 43(241): 109-126.

Goodman S.N., Zahurak M.L., Piantadosi S. (1995): Some practical improvements in the continual reassessment method for phase I studies. Statistics in Medicine 14(11): 1149-1161.

Korn E.L., Midthune D., Chen T.T., Rubinstein L.V., Christian M.C., Simon R.M. (1994): A comparison of two phase I trial designs. Statistics in Medicine 13(18): 1799-1806.

Le Tourneau C., Lee J.J., Siu L.L. (2009): Dose escalation methods in phase I cancer clinical trials. Journal of the National Cancer Institute 101(10): 708720 .

Leung D.H.Y., Wang Y.G. (2001): Isotonic designs for phase I trials. Controlled Clinical Trials 22(2): 126-138.

Lévy V., Zohar S., Porcher R., Chevret S. (2001): Alternate designs for conduct and analysis of phase I cancer trials. Blood 98(4): 1275-1275. 
O'Quigley J., Pepe M., Fisher L. (1990): Continual reassessment method: A practical design for phase I clinical trials in cancer. Biometrics 46(1): 33-48.

O'Quigley J., Shen L.Z. (1996): Continual reassessment method: A likelihood approach. Biometrics 52(2): 673-684.

O'Quigley J., Zohar S. (2006): Experimental designs for phase I and phase I/II dose-finding studies. British Journal of Cancer 94(5): 609-613.

Reiner E., Paoletti X., O'Quigley J. (1999): Operating characteristics of the standard phase I clinical trial design. Computational Statistics and Data Analysis 30(3): 303-315.

Simon R., Rubinstein L., Arbuck S.G., Christian M.C., Freidlin B., Collins J. (1997): Accelerated titration designs for phase I clinical trials in oncology. Journal of the National Cancer Institute 89(15): 1138-1147.

Storer B.E. (1989): Design and analysis of phase I clinical trials. Biometrics 45(3): 925-937. 